\title{
Metabolic approach for tumor delineation in glioma surgery: 3D MR spectroscopy image-guided resection
}

\author{
*Jie Zhang, MD,' Dong-Xiao Zhuang, MD, PhD, ${ }^{1}$ Cheng-Jun Yao, MD, PhD, ${ }^{1}$ Ching-Po Lin, PhD, ${ }^{2}$ \\ Tian-Liang Wang, BSc, ${ }^{3}$ Zhi-Yong Qin, MD, PhD, ${ }^{1}$ and Jin-Song Wu, MD, PhD' \\ ${ }^{1}$ Glioma Surgery Division, Department of Neurologic Surgery, Huashan Hospital, Shanghai Medical College, Fudan University, \\ Shanghai; ${ }^{2}$ Centre for Computational Systems Biology, Fudan University, Shanghai; and ${ }^{3}$ BrainLAB (Beijing) Medical Equipment \\ Trading Co., Ltd., Beijing, People's Republic of China
}

\begin{abstract}
OBJECTIVE The extent of resection is one of the most essential factors that influence the outcomes of glioma resection. However, conventional structural imaging has failed to accurately delineate glioma margins because of tumor cell infiltration. Three-dimensional proton MR spectroscopy ( $\left.{ }^{1} \mathrm{H}-\mathrm{MRS}\right)$ can provide metabolic information and has been used in preoperative tumor differentiation, grading, and radiotherapy planning. Resection based on glioma metabolism information may provide for a more extensive resection and yield better outcomes for glioma patients. In this study, the authors attempt to integrate 3D ${ }^{1} \mathrm{H}$-MRS into neuronavigation and assess the feasibility and validity of metabolically based glioma resection.
\end{abstract}

METHODS Choline (Cho)-N-acetylaspartate (NAA) index (CNI) maps were calculated and integrated into neuronavigation. The CNI thresholds were quantitatively analyzed and compared with structural MRI studies. Glioma resections were performed under $3 \mathrm{D}{ }^{1} \mathrm{H}-\mathrm{MRS}$ guidance. Volumetric analyses were performed for metabolic and structural images from a low-grade glioma (LGG) group and high-grade glioma (HGG) group. Magnetic resonance imaging and neurological assessments were performed immediately after surgery and 1 year after tumor resection.

RESULTS Fifteen eligible patients with primary cerebral gliomas were included in this study. Three-dimensional ${ }^{1} \mathrm{H}$ MRS maps were successfully coregistered with structural images and integrated into navigational system. Volumetric analyses showed that the differences between the metabolic volumes with different CNI thresholds were statistically significant $(p<0.05)$. For the LGG group, the differences between the structural and the metabolic volumes with $\mathrm{CNI}$ thresholds of 0.5 and 1.5 were statistically significant $(p=0.0005$ and 0.0129 , respectively). For the HGG group, the differences between the structural and metabolic volumes with $\mathrm{CNI}$ thresholds of 0.5 and 1.0 were statistically significant ( $p$ $=0.0027$ and 0.0497 , respectively). All patients showed no tumor progression at the 1-year follow-up.

CONCLUSIONS This study integrated 3D MRS maps and intraoperative navigation for glioma margin delineation. Optimum CNI thresholds were applied for both LGGs and HGGs to achieve resection. The results indicated that 3D ${ }^{1} \mathrm{H}-\mathrm{MRS}$ can be integrated with structural imaging to provide better outcomes for glioma resection.

http://thejns.org/doi/abs/10.3171/2015.6.JNS142651

KEY WORDS magnetic resonance spectroscopy; glioma; neuronavigation; surgical planning; oncology

$\mathrm{G}$ LIOMAS are the most common primary brain tumors, accounting for $80 \%$ of malignant central nervous system tumors, and can be categorized into 4 grades according to their biological behavior. Patients with low-grade gliomas (LGGs) typically survive more than 5 years. ${ }^{21}$ Those with high-grade gliomas (HGGs) have a survival time of approximately 1-3 years. An epidemiology study using primary brain tumor data collected from 1985 to 2005 showed that the 1-year survival rate of patients with malignant glioma is $53.9 \%$, while that of pa-

ABBREVIATIONS Cho = choline; $\mathrm{CNI}=$ choline-NAA index; $\mathrm{CSI}=$ chemical shift imaging; $\mathrm{DICOM}=$ digital imaging and communications in medicine; $\mathrm{DTI}=$ diffusion tensor imaging; $\mathrm{EOR}=$ extent of resection; $\mathrm{fMRI}=$ functional $\mathrm{MR}$ imaging; GTR = gross-total resection; ${ }^{1} \mathrm{H}-\mathrm{MRS}=$ proton $\mathrm{MR}$ spectroscopy; $\mathrm{HGG}=$ high-grade glioma; $\mathrm{LGG}=$ low-grade glioma; MPRAGE = magnetization-prepared rapid gradient-echo; NAA = N-acetylaspartate; OS = overall survival; $\mathrm{PFS}=$ progression-free survival; $\mathrm{PT}=$ pyramidal tract; ROI = region of interest; STR = subtotal resection; VOI = volume of interest.

SUBMITTED November 25, 2014. ACCEPTED June 18, 2015.

INCLUDE WHEN CITING Published online December 4, 2015; DOI: 10.3171/2015.6.JNS142651.

* Drs. Zhang and Zhuang contributed equally to this paper. 
tients with glioblastoma is only $30.3 \% .^{24}$ The prognostic factors for glioma include patient age, Karnofsky Performance Scale score, comorbidities, genetic status, and extent of resection (EOR). ${ }^{9}$ Much evidence shows that EOR is one of the most essential factors for progression-free survival (PFS) and overall survival (OS).

It is now well accepted that the principle of glioma surgery is maximal safe resection. Recently, Sanai et al. found that an EOR $\geq 78 \%$ impacts patient outcomes and that this trend continues even at the highest levels of resection. ${ }^{28}$ Another study showed that more aggressive resections of LGGs lead to a better prognosis with prolonged OS and PFS. ${ }^{4}$ Moreover, patients with glioblastoma multiforme have a better prognosis when the EOR $>98 \% .{ }^{17}$

Multimodal neuronavigation has become a useful tool in helping surgeons achieve maximal safe resections. According to the Response Assessment in Neuro-Oncology (RANO) standard, volumetric evaluation is based on structural MR images. ${ }^{37,39}$ For LGGs, T2-weighted or FLAIR imaging is used for volumetric assessment by delineating the hyperintense area. ${ }^{36}$ For HGGs, complete resection of enhancing lesions on T1-weighted images is considered gross-total resection (GTR). ${ }^{36,39}$ However, using traditional structural imaging to assess EOR can be misleading. For patients with lesions that exhibit the "typical" imaging features of astrocytoma, a 50\% false-positive rate has been obtained when using traditional structural imaging to predict a histological diagnosis..$^{15} \mathrm{~A}$ recent study demonstrated that infiltrating glioma cells often exceed the structural imaging boundaries for both LGGs and HGGs. ${ }^{12}$

Proton MR spectroscopy ('H-MRS) can identify chemical shifts in glioma cell metabolism and has been used in preoperative tumor differentiation, grading, and radiotherapy planning. ${ }^{3,22,33,35}$ It provides cellular metabolic information on tumors, including choline (Cho), $\mathrm{N}$-acetylaspartate (NAA), creatine (Cr), lactate, ${ }^{41}$ citrate, ${ }^{2}$ and lipid levels. In the past decade, this imaging modality has been widely used to differentiate tumors and glioma grades both ex vivo ${ }^{35}$ and in vivo. ${ }^{22}$ Moreover, metabolic images can predict PFS and OS better than anatomical images..$^{5,16,20}$ Some studies ${ }^{19,23}$ have shown that the ChoNAA index (CNI) can provide more reliable information on the extent of tumor infiltration. In a previous study, we examined the relationship between the CNI and glioma infiltration by using needle biopsies performed under the guidance of neuronavigation. ${ }^{12}$

Combining MRS information into a neuronavigational system may provide a new perspective for surgeons and metabolic boundaries for tumor resection. However, the poor tumor coverage and resolution of multivoxel MRS hinder the navigational application using MRS. Recent advanced 3D ${ }^{1} \mathrm{H}-\mathrm{MRS}$ can provide more brain coverage within an acceptable amount of clinical time. It thus opens a window to monitor tumor resection based on metabolic information. In this study, we developed a method of integrating $3 \mathrm{D}{ }^{1} \mathrm{H}-\mathrm{MRS}$ information into a neuronavigation system for tumor resection. Different thresholds of CNIs were applied to delineate the metabolic margins of LGGs and HGGs, and the feasibility of CNI-guided tumor resection was assessed, as were patient outcomes.

\section{Methods Patients}

Patients who had a diagnosis of cerebral glioma were eligible to participate in this study. Patients were excluded when histology indicated a tumor other than glioma. Patients with contraindications to MRI were also excluded. This study was undertaken at the Huashan Hospital in Shanghai, China, with approval from the Huashan Institutional Review Board.

\section{Magnetic Resonance Imaging}

Magnetic resonance imaging was performed 1 day before surgery using a 3.0-T iMRI suite (IMRIS) with a Siemens 8-channel head coil. The imaging protocols consisted of a 3D T1-weighted magnetization-prepared rapid gradient-echo (MPRAGE) sequence (TR $1.900 \mathrm{msec}$, TE $2.93 \mathrm{msec}$, flip angle $9^{\circ}$, matrix size $256 \times 215$, slice thickness $1 \mathrm{~mm}$, FOV $250 \times 219 \mathrm{~mm}$, acquisition averages 1) or a T2-FLAIR sequence (TR $9.00 \mathrm{msec}$, TE $96 \mathrm{msec}$, TI $2.500 \mathrm{msec}$, flip angle $150^{\circ}$, slice thickness $2 \mathrm{~mm}$, matrix size $256 \times 160$ ). Diffusion tensor imaging (DTI) sequences (TR $7.600 \mathrm{msec}$, TE $91 \mathrm{msec}$; slice thickness 3 $\mathrm{mm}$, slice space $0 \mathrm{~mm}$, matrix size $128 \times 128$, voxel size $1.8 \times 1.8 \times 3 \mathrm{~mm}^{3}$, directions 30 ) were acquired for fiber tracking. Functional MRI (fMRI) data were acquired using a single-shot echo-planar imaging (EPI) sequence (TR $3.000 \mathrm{msec}$, TE $30 \mathrm{msec}$, FOV $240 \times 240 \mathrm{~mm}$, matrix size $96 \times 96$, slice thickness $3 \mathrm{~mm}$ ) for language areas or motor areas when necessary. The raw data were exported into the digital imaging and communications in medicine (DICOM) format and processed using iPlan Cranial Version 3.0 (BrainLab).

\section{Three-Dimensional ${ }^{1} \mathrm{H}-\mathrm{MRS}$}

Three-dimensional MRS was performed using a pointresolved spectroscopy sequence (PRESS; TR $1.700 \mathrm{msec}$, TE $135 \mathrm{msec}$, slice thickness $15 \mathrm{~mm}$, phase encoding $16 \times$ $16 \times 16$, FOV $120 \times 120 \mathrm{~mm}$, flip angle $90^{\circ}$, scan resolution $10 \times 10 \times 10 \mathrm{~mm}$ ). Chemical shift imaging sequences were applied to achieve water suppression. The volume of interest (VOI) was positioned to avoid areas such as bone, subcutaneous lipids, and other areas of magnetic susceptibility to ensure that the quality of the MRS would not be affected. After 3D MRS acquisition, the raw data were transferred to a postprocessing workstation (Syngo MultiModality Workplace, Siemens AG).

\section{Data Processing}

The raw spectroscopy data were imported into a Siemens Syngo workstation (Fig. 1). The regional metabolite distributions of Cho and NAA and their ratios were computed inside the VOI with a resolution of $10 \mathrm{~mm}^{3}$. The CNIs were interpolated for each voxel inside the VOI to generate different CNI thresholds for metabolic delineation. Then a 3D chemical shift imaging (CSI) map was generated and transformed into a gray value map, which contained spatial and normalized metabolite Cho/NAA information. An anatomical T2-FLAIR or 3D MPRAGE sequence was scanned for fusion. Bold fMRI and DTI 


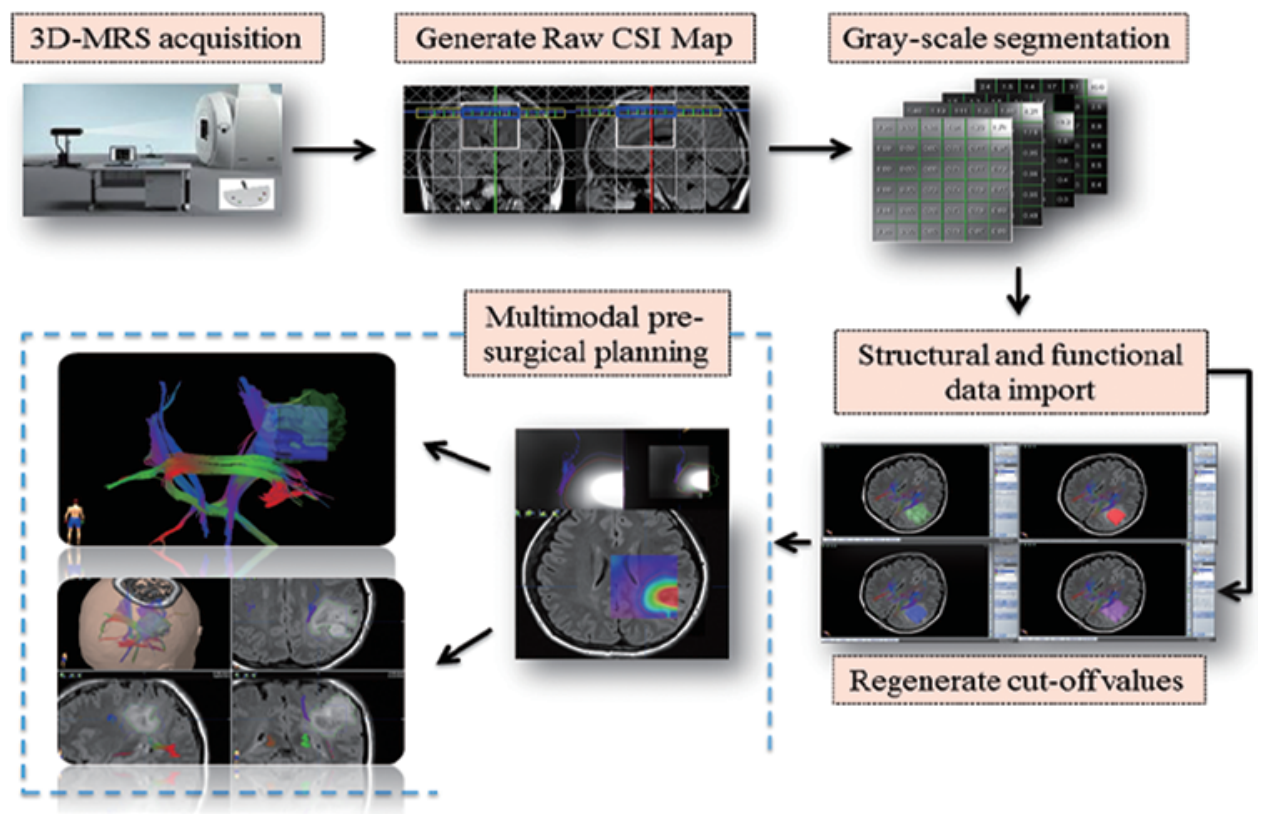

FIG. 1. Flowchart of registration of MRS metabolic image, DTI tractography, and structural image for volumetric tumor resection. Magnetic resonance imaging was performed using a 3.0-T iMRI suite. The raw CSI maps were generated on a Siemens Syngo workstation and transformed into gray value maps. All data were then transferred to the navigation plan software. The CNI contours were delineated and merged with structural and functional images. Figure is available in color online only.

data were prepared for merging. All of the raw data were saved in the DICOM format and transferred to iPlan Cranial 3.0 (BrainLab). In the iPlan software, the image data were fused using a frame of reference that was based on the anatomical images because all of the image data were acquired during the same session. The data processing procedure is demonstrated in a flowchart (Fig. 1). The accuracy of the fusion was verified using the SpyGlass and Edges functions for visual inspection. All of the data sets were fused and superimposed on anatomical images. For MRS, 3 different thresholds for CNIs $(0.5,1$, and 1.5$)$ were applied to contour the MRS margins. The gray scale maps were reconstructed using the rainbow color function and confirmed with previous pseudocolor maps that were generated in the Syngo system. Three different 3D MRS objects were created accordingly. For fiber tracking, the diffusion tensor image was overlaid on anatomical images. Then the region of interest (ROI) was defined (for example, the brainstem and the motor area were selected as the ROI for the lineate pyramidal tract [PT]). The fractional anisotropy threshold was set to $2.0-2.5$ to achieve the best tracking results. The results were merged and saved for surgical planning and navigation.

\section{Volumetric Analyses}

Three-dimensional MPRAGE images were used for the structural volumetric analyses. A GTR was defined as no tumor residuals identified on postoperative MRI, which consisted of T1-weighted contrast-enhanced images for HGGs and FLAIR images for LGGs, according to the RANO standard. A subtotal resection (STR) was defined as residual tumor $<10 \mathrm{~cm}^{3}$. Metabolic volumes were obtained according to the different CNIs and were outlined based on CSI maps. Manual segmentations were performed by a technician using iPlan software and were verified by a neurosurgeon. Additionally, the structural volumes were calculated within the MRS scanning bounding box using volumetric analysis for a better comparison. The data were further divided into 2 groups according to WHO grade: an LGG group including Grade II gliomas and an HGG group including Grades III and IV gliomas. The mixed-effects model for repeated measures was applied in the statistical analyses of tumor volumes, which were performed using SAS 9.2 (SAS Institute Inc.). A p value $\leq 0.05$ was considered statistically significant.

\section{Results}

From May to July 2013, 18 patients with brain gliomas were identified for 3D ${ }^{1} \mathrm{H}-\mathrm{MRS}$ scanning. Three cases were excluded from evaluation because the pathological diagnosis was metastasis. Therefore, 15 patients with primary cerebral gliomas were eligible for further analyses (Table 1).

Different CNI thresholds were applied to delineate the metabolic volumes. The volumetric assessments based on both structural and metabolic imaging are summarized in Tables 2 and 3. The differences between the metabolic volumes with different CNI thresholds were statistically significant $(\mathrm{p}<0.05)$. For the LGG group, the differences between the structural and the metabolic volumes with CNI thresholds of 0.5 and 1.5 were statistically significant $(\mathrm{p}=$ 0.0005 and $p=0.0129$, respectively). For the HGG group, the differences between the structural and metabolic volumes with CNI thresholds of 0.5 and 1.0 were statistically significant ( $p=0.0027$ and $p=0.0497$, respectively). For all of the patients, tumor resections were performed under 
TABLE 1. Demographics and clinical characteristics for 15 patients with cerebral glioma

\begin{tabular}{|c|c|}
\hline Variable & Value \\
\hline \multicolumn{2}{|l|}{ Age in yrs } \\
\hline Median & 46 \\
\hline Range & $32-71$ \\
\hline No. of males (\%) & $7(46.67)$ \\
\hline \multicolumn{2}{|l|}{ Clinical onset (no. [\%]) } \\
\hline Seizure & $7(46.67)$ \\
\hline Headache & $5(33.33)$ \\
\hline Language deficit & $1(6.67)$ \\
\hline Accidentally found & $2(13.33)$ \\
\hline \multicolumn{2}{|l|}{ Tumor location (no. [\%]) } \\
\hline Frontal lobe & $7(46.67)$ \\
\hline Parietal lobe & $4(26.67)$ \\
\hline Temporal lobe & $3(20.0)$ \\
\hline Insular & $1(6.67)$ \\
\hline \multicolumn{2}{|c|}{ Histological type, WHO tumor grade (no. [\%]) } \\
\hline Diffuse astrocytoma, II & $4(26.67)$ \\
\hline Oligodendroglioma, II & $4(26.67)$ \\
\hline Anaplastic astrocytoma, III & $1(6.67)$ \\
\hline Anaplastic oligodendroma, III & $2(13.33)$ \\
\hline Glioblastoma multiforme, IV & $4(26.67)$ \\
\hline \multicolumn{2}{|l|}{ Tumor vol in $\mathrm{cm}^{3}$} \\
\hline Median & 33.83 \\
\hline Range & $12.00-102.72$ \\
\hline \multicolumn{2}{|l|}{ GTR (no./total no. [\%]) } \\
\hline All grades & $13 / 15(86.67)$ \\
\hline Low grade (I or II) & $7 / 8(87.50)$ \\
\hline High grade (III or IV) & 6/7 (85.71) \\
\hline \multicolumn{2}{|l|}{ Postop deficit (no./total no. [\%]) } \\
\hline Short-term deficit & $1 / 15(6.7)$ \\
\hline Long-term deficit & $0 / 15(0)$ \\
\hline 1-year PFS (no./total no. [\%]) & $15 / 15(100)$ \\
\hline
\end{tabular}

the guidance of the 3D MRS and DTI results. The selection of the metabolic margin was based on the principle of achieving a maximal safe resection in individual patients. Postoperative MRI confirmed GTRs in 13 patients (Table 4). The average resected volume according to the metabolic margin was $59.48 \%$ greater than the structural volume. Only 1 patient suffered from a transient postoperative language deficit. No long-term motor deficits were found in any of the patients. At the 1-year follow-up, no tumor recurrence was found in the LGG group (Table 1). One patient from the HGG group was found to have a tumor recurrence at the 9-month follow-up.

\section{Illustrative Cases \\ Case 10}

This 50-year-old male was referred to our hospital and presented with a 1-year history of headache and weakness of the left limbs. He suffered from left facial paralysis 1 week before admission. The neurological examination revealed classic left central paralysis, and the muscle strength of the left limbs was Grade 4 (manual muscle testing). In addition, he had hyperactive deep tendon reflexes of the left lower extremities. A head MRI study showed a right frontal lesion with irregular contrast enhancement (Fig. 2A and C). The preoperative diagnosis was HGG. Diffusion tensor imaging was performed to trace the pyramidal tract (PT; Fig. 2C-F). Three-dimensional ${ }^{1} \mathrm{H}-\mathrm{MRS}$ revealed different metabolic margins that were generated for CNIs of $0.5,1.0$, and 1.5. Dice's coefficient between CNI 1.0 and structural volume showed a strong association in this case. Data were uploaded into iPlan 3.0 Cranial software for presurgical planning. Because a CNI of 1.0 was adjacent to the PT, while 0.5 overlapped with the PT, to achieve maximal safe resection, the EOR was carried as close as possible to the metabolic margin generated with a CNI of 1.0. A safe margin of $0.8 \mathrm{~mm}$ was set to avoid further neurological deficits even though this margin was near the PT (Fig. 2E and F). Ultimately, an STR was achieved (Fig. 2B). The patient suffered no neurological deficits postoperatively.

\section{Case 4}

This 33-year-old female came to our hospital with a 10-month history of repeated seizure attacks. She suffered no neurological deficits on admission. Magnetic resonance imaging showed a lesion located in the right parietal lobe. The lesion was not contrast-enhanced by gadolinium. Diffusion tensor imaging and 3D ${ }^{1} \mathrm{H}-\mathrm{MRS}$ were scheduled 1 day before her operation. The metabolic margins were delineated and superimposed on structural images (Fig. $3 \mathrm{~A})$. Fiber tracking revealed the relationship between the

TABLE 2. Summary of volumetric assessment results based on structural and metabolic imaging*

\begin{tabular}{|c|c|c|c|c|c|}
\hline \multirow[b]{3}{*}{ Variable } & \multirow{3}{*}{$\begin{array}{c}\text { No. of } \\
\text { Patients }\end{array}$} & \multicolumn{4}{|c|}{ Mean Tumor Vol in $\mathrm{cm}^{3}$ (range) } \\
\hline & & \multirow{2}{*}{$\begin{array}{l}\text { Structural } \\
\text { Imaging }\end{array}$} & \multicolumn{3}{|c|}{ MRS CNI } \\
\hline & & & 0.5 & 1.0 & 1.5 \\
\hline LGG & 8 & 47.99 (23.04-98.82) & $79.58 \dagger(39.36-141.31)$ & $41.45(22.08-75.26)$ & $23.66 \dagger(3.84-41.47)$ \\
\hline HGG & 7 & $42.74(12.00-102.72)$ & $106.27 \dagger(59.39-187.20)$ & $74.29+(22.02-152.64)$ & $48.97(8.70-113.28)$ \\
\hline Total & 15 & 45.54 (12.00-102.72) & $92.04(39.36-187.20)$ & 56.77 (22.02-152.64) & $35.47(3.84-113.28)$ \\
\hline
\end{tabular}


TABLE 3. A volumetric comparison of the structural imaging results with different CNIs

\begin{tabular}{cccccc}
\hline CNI Cutoff Values & Estimated Mean Value & Lower Limit of Range & Upper Limit of Range & t Value & $p$ Value \\
\hline LGG & & & & & \\
\hline Vs 0.5 & $-31.59 \pm 12.47$ & -44.06 & -19.13 & -5.99 & 0.0005 \\
\hline Vs 1.0 & $+6.55 \pm 12.97$ & -6.43 & 19.52 & 1.19 & 0.2719 \\
\hline Vs 1.5 & $+24.33 \pm 17.37$ & 6.96 & 41.70 & 3.31 & 0.0129 \\
\hline HGG & & & & & 0.0027 \\
\hline Vs 0.5 & $-63.53 \pm 31.73$ & -95.26 & -31.80 & -4.90 & 0.0497 \\
\hline Vs 1.0 & $-31.55 \pm 31.49$ & -63.04 & -0.066 & -2.45 & 0.5527 \\
\hline Vs 1.5 & $-6.23 \pm 24.24$ & -30.47 & 18.01 & -0.63 & \\
\hline
\end{tabular}

metabolic margins and the PT (Fig. 3C-F). The margin with a CNI of 0.5 overlapped the PT (Fig. 3D and E). A resection based on a margin of 0.5 would most likely have caused postoperative motor deficits (Fig. 3F). For areas adjacent to the PT, a CNI margin of 1.0 was applied for tumor resection (Fig. 3C and D). Dice's coefficient of the volumes in this case indicated a strong correlation between the CNI 1.0 margin and the structural margin. For noneloquent areas, a CNI margin of 0.5 was applied for resection (Fig. 3B). An STR was achieved based on the intraoperative MRI studies. There were no neurological deficits in this patient.

\section{Discussion}

In the present study we aimed to perform metabolically based tumor resection by integrating neuronavigation data with 3D ${ }^{1} \mathrm{H}-\mathrm{MRS}$, which can detect metabolic abnormalities confirmed by biopsy diagnosis of gliomas. ${ }^{24}$ Three different thresholds of CNIs were established to delineate the metabolic margins for either HGG or LGG resection. Taking eloquent areas into consideration, one can apply the most suitable CNI-based tumor boundary to achieve a maximal safe resection as well as avoid potential neurological deficits. By adopting this method, we found no tumor recurrence in our LGG group and only 1 patient from the HGG group who had a tumor recurrence after the 1-year follow-up. Our results indicate that tumor resection with 3D ${ }^{1} \mathrm{H}-\mathrm{MRS}$-aided navigation is safe and feasible for maximal safe resection.

\section{Metabolic Index for Tumor Margin Delineation and Presurgical Planning}

Conventionally, glioma resections are based on tumor margins delineated using structural T1 and T2-FLAIR MR images. However, the benefits gained from navigational resections are limited, with a poor prognosis even in the GTR patients. It is now clear that glioma cells far exceed the margins revealed on traditional structural images. Proton $\left({ }^{\circ} \mathrm{H}\right)$ MRS has shown its ability to detect metabolic abnormalities where conventional MRI cannot make such distinctions. ${ }^{7}$ Previous studies have found that

TABLE 4. Detailed information on metabolic resection

\begin{tabular}{|c|c|c|c|c|c|c|c|c|}
\hline \multirow{2}{*}{$\begin{array}{l}\text { Case No./Sex/Age } \\
\text { at Admission (yrs) }\end{array}$} & \multirow{2}{*}{$\begin{array}{l}\text { Presenting } \\
\text { Symptom }\end{array}$} & \multirow[b]{2}{*}{ Tumor Location } & \multirow{2}{*}{$\begin{array}{c}\text { WHO Glioma } \\
\text { Grade }\end{array}$} & \multirow{2}{*}{$\begin{array}{l}\text { Structural } \\
\text { Vol }\left(\mathrm{cm}^{3}\right)\end{array}$} & \multicolumn{3}{|c|}{ Metabolic Vol $\left(\mathrm{cm}^{3}\right)$} & \multirow[b]{2}{*}{ Postop Cavity $\left(\mathrm{cm}^{3}\right)$} \\
\hline & & & & & 1.5 & 1.0 & 0.5 & \\
\hline $1 / \mathrm{M} / 33$ & Headache & Lt temporal & III & 12.00 & 37.60 & 60.00 & 82.40 & 79.28 \\
\hline $2 / F / 58$ & Seizure attack & Rt frontal & IV & 28.80 & 66.40 & 107.20 & 145.60 & 48.78 \\
\hline $3 / M / 42$ & Seizure attack & Rt parietal & II & 56.64 & 3.84 & 22.08 & 97.92 & 60.61 \\
\hline $4 / F / 33^{*}$ & Seizure attack & Rt parietal & $\|$ & 27.84 & 29.76 & 39.36 & 82.56 & 24.99 \\
\hline $5 / \mathrm{M} / 37$ & Incidental & Lt frontal & ॥ & 98.82 & 41.47 & 75.26 & 141.31 & 107.69 \\
\hline $6 / F / 60$ & Incidental & Rt parietal & III & 19.46 & 8.70 & 32.26 & 69.63 & 24.45 \\
\hline 7/M/47 & Headache & Rt insular & IV & 102.72 & 113.28 & 152.64 & 187.20 & 115.78 \\
\hline $8 / \mathrm{M} / 71$ & Aphasia & Lt frontal & IV & 58.56 & 35.52 & 53.76 & 80.64 & 65.72 \\
\hline 9/F/56 & Seizure & Rt frontal & $\|$ & 56.64 & 36.48 & 58.56 & 82.56 & 58.88 \\
\hline $10 / \mathrm{M} / 50^{*}$ & Headache & Rt frontal & IV & 41.28 & 71.04 & 92.16 & 119.04 & 36.33 \\
\hline $11 / F / 58$ & Seizure & Lt frontal & II & 28.80 & 15.36 & 35.52 & 65.28 & 54.39 \\
\hline 12/F/39 & Seizure & Lt parietal & II & 26.88 & 13.44 & 22.08 & 39.36 & 29.82 \\
\hline 13/M/37 & Headache & Rt temporal & II & 23.04 & 11.52 & 22.08 & 49.92 & 44.50 \\
\hline $14 / F / 46$ & Seizure & Rt frontal & III & 36.35 & 10.24 & 22.02 & 59.39 & 36.49 \\
\hline $15 / F / 32$ & Headache & Rt temporal & II & 65.28 & 37.44 & 56.64 & 77.76 & 78.65 \\
\hline
\end{tabular}

* Gross-total resection could not be achieved in Cases 4 and 10 because of the invasion of PTs. 

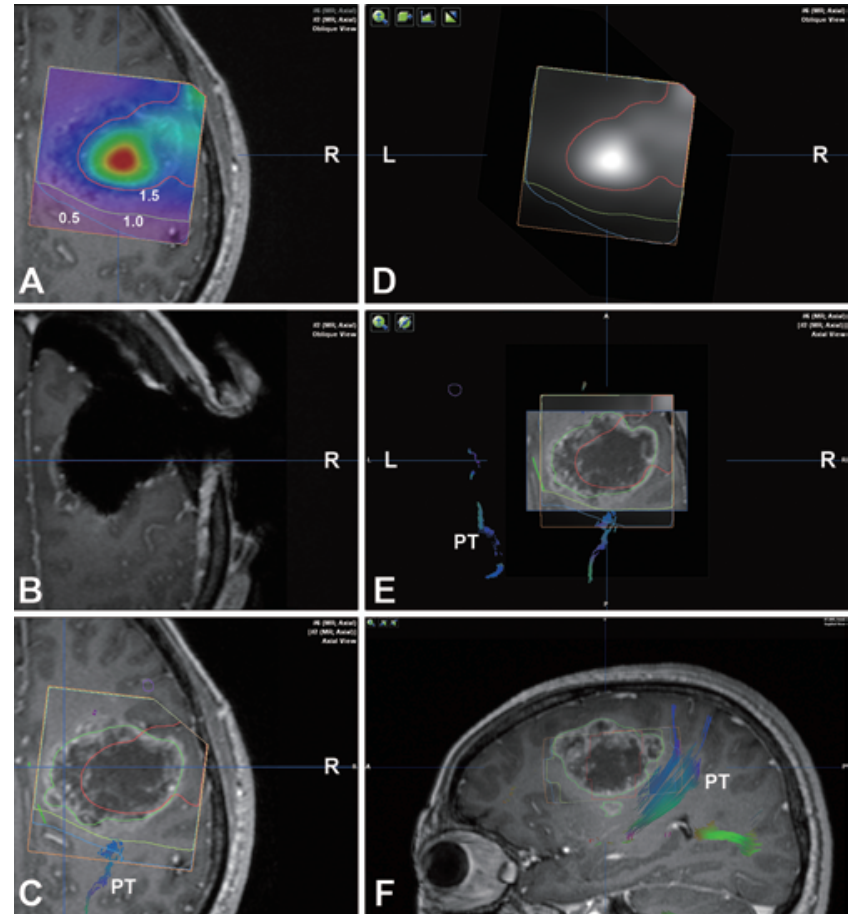

FIG. 2. Case 10. The process of converting and merging $3 D{ }^{1} H-M R S$ images with structural images. A: 3D ${ }^{1} \mathrm{H}-\mathrm{MRS}$ revealed different metabolic margins generated for CNIs of $0.5,1.0$, and 1.5 , which were transferred into iPlan 3.0 Cranial. The pseudocolor maps were then generated. B: Postoperative MR image. C: Functional MRI and DTI located the hand motor areas and PTs. D and E: The contour line for a CNI of 1.0 was adjacent to the PTs. Red: 1.5; yellow: 1.0; and blue: 0.5 . To achieve a maximal safe resection, the EOR was carried as close as possible to the metabolic margin generated for a CNI of 1.0. F: Sagittal view revealing the relationship of the PTs and metabolic margins. A safe margin of $0.8 \mathrm{~mm}$ was set to avoid further neurological deficits even though this margin was near the PTs. An STR was achieved in this patient. Figure is available in color online only.

gliomas tend to have decreased NAA and creatine and elevated Cho levels compared with levels in normal brain tissue. ${ }^{12,22,23,42}$ Yang et al. also found that there were statistically significant differences in CNIs between the HGGs and LGGs. ${ }^{42}$ In their study, the mean CNI was $5.9 \pm 2.62$ for HGGs and $1.65 \pm 1.37$ for LGGs. In a previous study, we performed needle biopsies to extract different tissue samples in a patient series according to different CNI indexes. ${ }^{12}$ Immunohistochemical staining was performed to identify the tumor cell infiltration, and the corresponding threshold ratios of CNIs were calculated. Our results indicated that CNI thresholds of $0.5,1.0$, and 1.5 can predict tumor infiltration with probabilities of $38 \%, 60 \%$, and $79 \%$ in HGGs and $16 \%, 39 \%$, and $67 \%$ in LGGs, respectively. According to these analyses, we applied different CNIs to delineate the metabolite-based glioma margins for tumor resection in the present study. We showed that different CNI margins can be used for HGGs and LGGs to achieve an individualized tumor resection strategy. Further volumetric analyses revealed that the metabolic volumes are larger than the structural volumes under a CNI of 0.5 , and this difference is statistically significant. For HGGs,
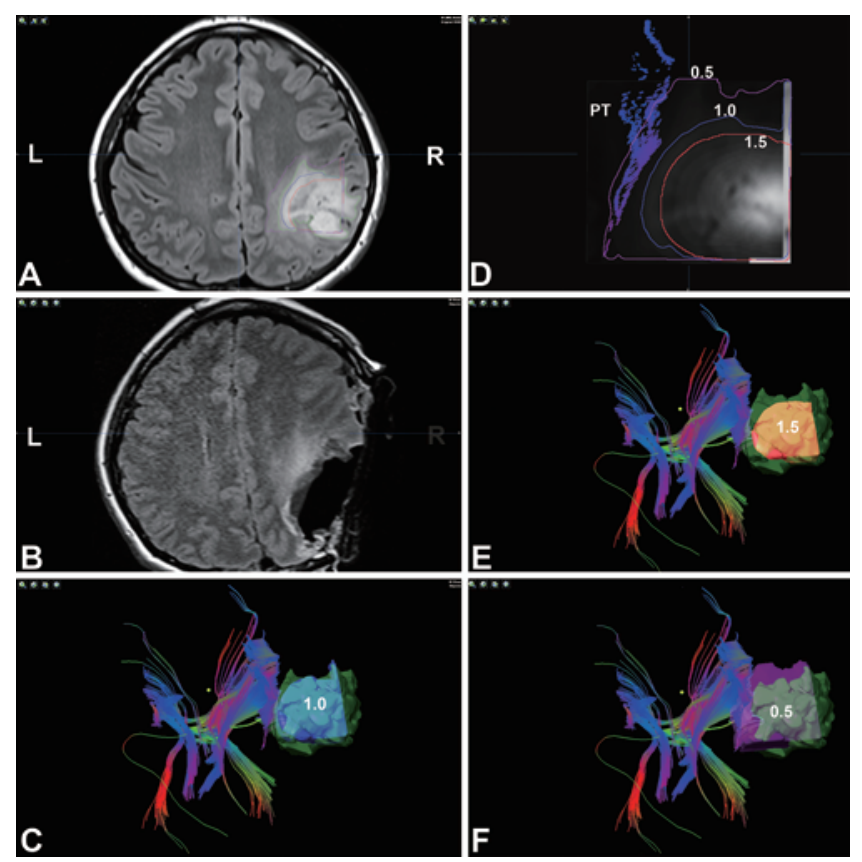

FIG. 3. Case 4. Metabolic maps for LGG. A: Different MRS contour lines were generated for an LGG resection; the metabolic margins were delineated and superimposed on structural images. B: For noneloquent area, a CNI margin of 0.5 was applied for tumor resection, and a margin of 1.0 was applied when near the PT to preserve motor functions. An STR was achieved based on intraoperative MRI. C: A safer resection could be achieved with a CNI contour line of 1.0. D: Fiber tracking revealed the relationship between the metabolic margins and the PT, which revealed the relationship among different $\mathrm{CNI}$ contour lines and the PT. E and F: A CNI contour line of 0.5 better delineated the tumor region; however, the margin overlapped the PT in this case. A resection based on a margin of 0.5 would most likely have caused postoperative motor deficits. Figure is available in color online only.

CNI thresholds of 1.0 and 0.5 both exceeded the structural margins defined by contrast-enhancing boundaries $(\mathrm{p}<$ 0.05 ). Therefore, a CNI of 1.0 can be used for navigation to achieve the same effect as structural margins in LGGs. The margin with a CNI of 0.5 can be selected to perform an aggressive resection. For HGGs, the margins with CNIs of 1.0 and 0.5 can both be used for further resection. Accordingly, our 2 cases with glioma invading eloquent areas demonstrated that metabolic imaging can provide various choices of metabolic margins based on their spatial relationship with eloquent areas. Previous DTI studies have indicated that a safe margin of $8 \mathrm{~mm}$ should be maintained between the resection margin and the PTs ${ }^{40,45}$ Our results also indicated that a well-planned individualized surgical strategy can be implemented by the integration of MRS data and functional mapping and structural margins. Surgeons sometimes have to adjust the surgical plan based on functional mapping, either from direct electrical stimulation (DES) or neuroimaging. For tumors invading eloquent cortex, preoperative task-related fMRI and DTI can be performed. Intraoperative language and/or motor cortical stimulations can also be performed to identify eloquent sites. A 10-mm margin from a DES-positive site was preserved during tumor resection to avoid permanent 
functional deficits. However, preoperative task-related BOLD fMRI showed a variety of sensitivities and specificities because of numerous factors such as patient status and the selection of the functional stimulus. ${ }^{1,10}$ Awake surgery, which is deemed a gold standard for functional mapping, may also be subject to the limited selection of intraoperative tasks. Plenty of studies have shown that the short-term functional deficit rates after awake surgery are higher than those with general anesthesia, although the patients showed better recovery in the long-term. ${ }^{6,8,27}$

\section{Metabolic Versus Structural Resection for Gliomas}

Previous studies have applied proton MRS for imageguided biopsies and postoperative radiotherapies. ${ }^{25,29,33}$ However, no postoperative outcomes for proton MRSguided tumor resections have been reported. In this study, GTRs or even supratotal resections were performed in 13 of 15 patients. After surgery, all patients received standard radio- and chemotherapies according to their WHO glioma grades. At the 1-year follow-up, no tumor progression was found in the LGG and HGG groups. From the Surveillance, Epidemiology, and End Results (SEER) program, Porter et al. have found that the 1-year survival rate for malignant glioma, LGG, and $\mathrm{HGG}$ patients is $50.4 \%-$ $57.3 \%, 68.2 \%-93.4 \%$, and $30.3 \%-77.8 \%$, respectively. ${ }^{24}$ Keles et al. also found that the mean glioma progression time is 13.3 months in GTR patients and 11.5 months in STR patients. ${ }^{13}$ In our study, no progression was found at the 12-month follow-up. This may result from the metabolic resections beyond the margin of GTR in our patients. A systematic review on glioma EOR and survival revealed that more extensive resections may be linked to a more favorable life expectancy for both LGG and HGG patients. ${ }^{26}$ Despite the better survival rate presented in our study, a well-designed clinical trial with large case numbers is needed to draw further conclusions.

\section{Techniques for Navigational Integration of 3D ${ }^{1} \mathrm{H}-\mathrm{MRS}$}

The 3D ${ }^{1} \mathrm{H}-\mathrm{MRS}$ data were scanned under a 3.0-T iMRI operating suite. Compared with single-voxel MRS and multivoxel 2D ${ }^{1} \mathrm{H}-\mathrm{MRS}, 3 \mathrm{D}{ }^{1} \mathrm{H}-\mathrm{MRS}$ can provide more spatial coverage of multiple tumor regions. A previous study has shown that the sensitivity, specificity, and diagnostic accuracy of 3D ${ }^{1} \mathrm{H}-\mathrm{MRS}$ were $94.1 \%, 100 \%$, and $96.2 \%$, respectively. ${ }^{44}$ Stadlbauer et al. have attempted to integrate 2D ${ }^{1} \mathrm{H}$-MRS into a neuronavigation system. They replaced the raw data of the anatomical slices with processed MRI/MRS hybrid data sets and successfully performed biopsies in all patients. ${ }^{32}$ A recent study by Ken et al. proposed a method of integrating 3D MRS into a treatment planning system for radiation therapy. ${ }^{14}$ The red-green-blue snapshots of anatomical-metabolic images were processed for coregistration. In our study, several attempts were made to integrate CNI with the navigational system. One approach was to use third-party free software (Sivic, Tarquin, and so forth) to perform MRS analysis before integration. However, these software packages did not support the Siemens 3D MRS data sets. Finally, we tried to integrate 3D ${ }^{1} \mathrm{H}-\mathrm{MRS}$ into a neuronavigation system by generating gray scale maps with DICOM information (Fig. 1). Our method of integration may have several advantages: 1) postprocessing of MRS data is more convenient because the coregistration of metabolic and structural images can be performed automatically; 2) data obtained using the other modalities such as DTI and fMRI can be easily coregistered as the original structural images remain intact; and 3) multiple metabolic margins can be presented at the same time for tumor resection guidance.

\section{Limitations and Perspectives}

Given the nature of metabolic imaging, the spatial resolution of MRS images is lower than that of structural images. Higher magnetic intensity may provide better signal quality and imaging resolution. Magnetic resonance spectroscopy signal is poor when tumors are located near convex or ventricular systems, which could result in falsepositive high CNIs in normal brain tissue and in tissue near the cranial bone. This problem can be partially overcome by using water suppression in specific regions and carefully selecting margins based on MRS. Another issue is that this technique can only be applied on the BrainLab navigational system at this time. As far as we know, the 3D MRS data sets do not work well on other navigation modalities such as Medtronic or with third-party software, partly because of the different scanning parameters between anatomical slices and spectroscopy slices. A more generalized and user-friendly module for 3D MRS integration is needed for future study. Meanwhile, other metabolites, such as myoinositol, ${ }^{31}$ citrate, $^{2}$ phosphorylcholine, and lactate may also be applied for tumor margin delineation. ${ }^{34}$ In addition, newly developed technologies, such as phosphorus MRS (P-MRS) ${ }^{18,37}$ and whole-brain proton MRS, ${ }^{11}$ may also contribute to the delineation of tumor margins in future surgery.

In current practice, although there is no Class I evidence that the EOR improves OS in glioma patients, maximum resection as far as is feasible is still recommended for both LGGs $^{30}$ and HGGs. ${ }^{38}$ The current standard for total glioma resection is solely based on structural images. For LGGs, because of the gradual growth of the tumor, the brain may adapt and reorganize function as the tumor infiltrates. ${ }^{43}$ In this study, we demonstrated the feasibility of integrating 3D MRS and neuronavigation and proved that metabolic resection is feasible and may provide a better prognosis than structural tumor resection alone. We have applied various CNIs to delineate the glioma metabolic margins and found that these margins exceeded the structural margins with a CNI less than 1.0 in LGGs and 1.5 in HGGs. Since CNI is highly correlated with glioma cell infiltration, the metabolic margin may be valuable information for the EOR.

\section{Conclusions}

In this study, glioma margin delineation using $3 \mathrm{D}{ }^{1} \mathrm{H}-$ MRS was presented and integrated with neuronavigation for metabolic glioma resection. The results showed that optimal CNI thresholds can be applied for LGG and HGG resection. The 1-year follow-up indicated that glioma resection based on 3D ${ }^{1} \mathrm{H}-\mathrm{MRS}$ may contribute to a better prognosis. Taking eloquent areas into consideration, we determined that the most suitable CNI-based tumor 
boundary can be applied to avoid potential neurological deficits. Combining 3D ${ }^{1} \mathrm{H}-\mathrm{MRS}$, fMRI, and DTI, the maximal safe resection for gliomas can be defined as follows: for noneloquent areas, the minimal CNI threshold of 0.5 could be used for maximal tumor resection; for tumors near eloquent areas, a safe margin of $8 \mathrm{~mm}$ should be used to reduce postoperative deficits. Further high-quality randomized control studies are suggested to confirm the outcomes of metabolic glioma resection.

\section{Acknowledgments}

We thank Zhong Yang for the 3D-MRS image data sets acquisition; Uli Mezger for technical support on MRS data transformation; Jian-bing Shi for surgical neuronavigation merge; and Yan-yan Song for statistical analyses. This work was funded by the National Natural Science Foundation of China (Project Nos. 81071117 \& 81171295), the National Key Technology R\&D Program of China (No. 2014BAI04B05), and Shanghai Municipal Health Bureau (XBR2011022).

\section{References}

1. Bennett CM, Miller MB: How reliable are the results from functional magnetic resonance imaging? Ann N Y Acad Sci 1191:133-155, 2010

2. Blüml S, Panigrahy A, Laskov M, Dhall G, Krieger MD, Nelson $\mathrm{MD}$, et al: Elevated citrate in pediatric astrocytomas with malignant progression. Neuro Oncol 13:1107-1117, 2011

3. Bulik M, Jancalek R, Vanicek J, Skoch A, Mechl M: Potential of MR spectroscopy for assessment of glioma grading. Clin Neurol Neurosurg 115:146-153, 2013

4. Capelle L, Fontaine D, Mandonnet E, Taillandier L, Golmard JL, Bauchet L, et al: Spontaneous and therapeutic prognostic factors in adult hemispheric World Health Organization Grade II gliomas: a series of 1097 cases: clinical article. J Neurosurg 118:1157-1168, 2013

5. Carpinelli G, Carapella CM, Palombi L, Raus L, Caroli F, Podo F: Differentiation of glioblastoma multiforme from astrocytomas by in vitro $1 \mathrm{H}$ MRS analysis of human brain tumors. Anticancer Res 16 (3B):1559-1563, 1996

6. De Witt Hamer PC, Robles SG, Zwinderman A, Duffau H, Berger MS: Impact of intraoperative stimulation brain mapping on glioma surgery outcome: a meta-analysis. J Clin Oncol 30:2559-2565, 2012

7. Dowling C, Bollen AW, Noworolski SM, McDermott MW, Barbaro NM, Day MR, et al: Preoperative proton MR spectroscopic imaging of brain tumors: correlation with histopathologic analysis of resection specimens. AJNR Am J Neuroradiol 22:604-612, 2001

8. Duffau H, Lopes M, Arthuis F, Bitar A, Sichez JP, Van Effenterre R, et al: Contribution of intraoperative electrical stimulations in surgery of low grade gliomas: a comparative study between two series without (1985-96) and with (1996-2003) functional mapping in the same institution. J Neurol Neurosurg Psychiatry 76:845-851, 2005

9. Eyüpoglu IY, Buchfelder M, Savaskan NE: Surgical resection of malignant gliomas-role in optimizing patient outcome. Nat Rev Neurol 9:141-151, 2013

10. Giussani C, Roux FE, Ojemann J, Sganzerla EP, Pirillo D, Papagno C: Is preoperative functional magnetic resonance imaging reliable for language areas mapping in brain tumor surgery? Review of language functional magnetic resonance imaging and direct cortical stimulation correlation studies. Neurosurgery 66:113-120, 2010

11. Govind V, Gold S, Kaliannan K, Saigal G, Falcone S, Arheart $\mathrm{KL}$, et al: Whole-brain proton MR spectroscopic imaging of mild-to-moderate traumatic brain injury and correlation with neuropsychological deficits. J Neurotrauma 27:483-496, 2010

12. Guo J, Yao C, Chen H, Zhuang D, Tang W, Ren G, et al: The relationship between Cho/NAA and glioma metabolism: implementation for margin delineation of cerebral gliomas. Acta Neurochir (Wien) 154:1361-1370, 2012

13. Keles GE, Anderson B, Berger MS: The effect of extent of resection on time to tumor progression and survival in patients with glioblastoma multiforme of the cerebral hemisphere. Surg Neurol 52:371-379, 1999

14. Ken S, Vieillevigne L, Franceries X, Simon L, Supper C, Lotterie JA, et al: Integration method of 3D MR spectroscopy into treatment planning system for glioblastoma IMRT dose painting with integrated simultaneous boost. Radiat Oncol 8:1, 2013

15. Kondziolka D, Lunsford LD, Martinez AJ: Unreliability of contemporary neurodiagnostic imaging in evaluating suspected adult supratentorial (low-grade) astrocytoma. J Neurosurg 79:533-536, 1993

16. Kousi E, Tsougos I, Tsolaki E, Fountas KN, Theodorou K, Fezoulidis I, et al: Spectroscopic evaluation of glioma grading at 3T: the combined role of short and long TE. ScientificWorldJournal 2012:546171, 2012

17. Lacroix M, Abi-Said D, Fourney DR, Gokaslan ZL, Shi W, DeMonte F, et al: A multivariate analysis of 416 patients with glioblastoma multiforme: prognosis, extent of resection, and survival. J Neurosurg 95:190-198, 2001

18. Laxer KD: Phosphorus MRS: pH, ATP, PCr. Adv Neurol 83:273-277, 2000

19. Lee PL, Gonzalez RG: Magnetic resonance spectroscopy of brain tumors. Curr Opin Oncol 12:199-204, 2000

20. Li Y, Lupo JM, Parvataneni R, Lamborn KR, Cha S, Chang SM, et al: Survival analysis in patients with newly diagnosed glioblastoma using pre- and postradiotherapy MR spectroscopic imaging. Neuro Oncol 15:607-617, 2013

21. Louis DN, Ohgaki H, Wiestler OD, Cavenee WK, Burger PC, Jouvet A, et al: The 2007 WHO classification of tumours of the central nervous system. Acta Neuropathol 114:97-109, 2007

22. McKnight TRLK, Lamborn KR, Love TD, Berger MS, Chang S, Dillon WP, et al: Correlation of magnetic resonance spectroscopic and growth characteristics within Grades II and III gliomas. J Neurosurg 106:660-666, 2007

23. Oshiro S, Tsugu H, Komatsu F, Abe H, Onishi H, Ohmura T, et al: Quantitative assessment of gliomas by proton magnetic resonance spectroscopy. Anticancer Res 27 (6A):3757-3763, 2007

24. Porter KR, McCarthy BJ, Berbaum ML, Davis FG: Conditional survival of all primary brain tumor patients by age, behavior, and histology. Neuroepidemiology 36:230-239, 2011

25. Qu JR, Jiang T, Dai JP, Li HL, Luo JP, Li SW, et al: Proton magnetic resonance spectroscopy predicts radiotherapy response and time-to-progression in high-grade gliomas after surgery. Chin Med J (Engl) 125:4334-4337, 2012

26. Sanai N, Berger MS: Glioma extent of resection and its impact on patient outcome. Neurosurgery 62:753-764, 264266, 2008

27. Sanai N, Mirzadeh Z, Berger MS: Functional outcome after language mapping for glioma resection. N Engl J Med 358: $18-27,2008$

28. Sanai N, Polley MY, McDermott MW, Parsa AT, Berger MS: An extent of resection threshold for newly diagnosed glioblastomas. J Neurosurg 115:3-8, 2011

29. Shen G, Xu L, Xu M, Geng M, Tan Y, Li F: 1H-MR spectroscopy guided gamma knife radiosurgery for treatment of glioma. Turk Neurosurg 22:690-694, 2012

30. Soffietti R, Baumert BG, Bello L, von Deimling A, Duffau $\mathrm{H}$, Frénay M, et al: Guidelines on management of low-grade 
gliomas: report of an EFNS-EANO Task Force. Eur J Neurol 17:1124-1133, 2010

31. Srinivasan R, Phillips JJ, Vandenberg SR, Polley MY, Bourne $\mathrm{G}, \mathrm{Au} \mathrm{A}$, et al: Ex vivo MR spectroscopic measure differentiates tumor from treatment effects in GBM. Neuro Oncol 12:1152-1161, 2010

32. Stadlbauer A, Moser E, Gruber S, Nimsky C, Fahlbusch R, Ganslandt O: Integration of biochemical images of a tumor into frameless stereotaxy achieved using a magnetic resonance imaging/magnetic resonance spectroscopy hybrid data set. J Neurosurg 101:287-294, 2004

33. Stadlbauer A, Nimsky C, Buslei R, Pinker K, Gruber S, Hammen T, et al: Proton magnetic resonance spectroscopic imaging in the border zone of gliomas: correlation of metabolic and histological changes at low tumor infiltration-initial results. Invest Radiol 42:218-223, 2007

34. Venkatesh HS, Chaumeil MM, Ward CS, Haas-Kogan DA, James CD, Ronen SM: Reduced phosphocholine and hyperpolarized lactate provide magnetic resonance biomarkers of $\mathrm{PI} 3 \mathrm{~K} / \mathrm{Akt} / \mathrm{mTOR}$ inhibition in glioblastoma. Neuro Oncol 14:315-325, 2012

35. Vettukattil R, Gulati M, Sjøbakk TE, Jakola AS, Kvernmo NA, Torp SH, et al: Differentiating diffuse World Health Organization grade II and IV astrocytomas with ex vivo magnetic resonance spectroscopy. Neurosurgery 72:186-195, 2013

36. Vogelbaum MA, Jost S, Aghi MK, Heimberger AB, Sampson JH, Wen PY, et al: Application of novel response/progression measures for surgically delivered therapies for gliomas: Response Assessment in Neuro-Oncology (RANO) Working Group. Neurosurgery 70:234-244, 2012

37. Warnke PC, Kreth FW, Ostertag CB: Early postoperative magnetic resonance imaging after resection of malignant glioma: objective evaluation of residual tumor and its influence on regrowth and prognosis. Neurosurgery 36:872-874, 1995

38. Weller M, van den Bent M, Hopkins K, Tonn JC, Stupp R, Falini A, et al: EANO guideline for the diagnosis and treatment of anaplastic gliomas and glioblastoma. Lancet Oncol 15:e395-e403, 2014

39. Wen PY, Macdonald DR, Reardon DA, Cloughesy TF, Sorensen AG, Galanis E, et al: Updated response assessment criteria for high-grade gliomas: response assessment in neuro-oncology working group. J Clin Oncol 28:1963-1972, 2010

40. Wu JS, Zhou LF, Tang WJ, Mao Y, Hu J, Song YY, et al: Clinical evaluation and follow-up outcome of diffusion tensor imaging-based functional neuronavigation: a prospective, controlled study in patients with gliomas involving pyramidal tracts. Neurosurgery 61:935-949, 2007
41. Yamasaki F, Kurisu K, Kajiwara Y, Watanabe Y, Takayasu T, Akiyama Y, et al: Magnetic resonance spectroscopic detection of lactate is predictive of a poor prognosis in patients with diffuse intrinsic pontine glioma. Neuro Oncol 13:791801, 2011

42. Yang D, Korogi Y, Sugahara T, Kitajima M, Shigematsu Y, Liang L, et al: Cerebral gliomas: prospective comparison of multivoxel 2D chemical-shift imaging proton MR spectroscopy, echoplanar perfusion and diffusion-weighted MRI. Neuroradiology 44:656-666, 2002

43. Yordanova YN, Moritz-Gasser S, Duffau H: Awake surgery for WHO Grade II gliomas within "noneloquent" areas in the left dominant hemisphere: toward a "supratotal" resection. Clinical article. J Neurosurg 115:232-239, 2011

44. Zeng QS, Li CF, Zhang K, Liu H, Kang XS, Zhen JH: Multivoxel 3D proton MR spectroscopy in the distinction of recurrent glioma from radiation injury. J Neurooncol 84:63-69, 2007

45. Zhu FP, Wu JS, Song YY, Yao CJ, Zhuang DX, Xu G, et al: Clinical application of motor pathway mapping using diffusion tensor imaging tractography and intraoperative direct subcortical stimulation in cerebral glioma surgery: a prospective cohort study. Neurosurgery 71:1170-1184, 2012

\section{Disclosures}

The authors report no conflict of interest concerning the materials or methods used in this study or the findings specified in this paper.

\section{Author Contributions}

Conception and design: Yao, Zhuang, Qin, Wu. Acquisition of data: Zhang, Wang. Analysis and interpretation of data: Zhang, Lin, Wang. Drafting the article: Zhang, Zhuang, Qin. Critically revising the article: Yao, Zhang, Zhuang, Lin, Wu. Reviewed submitted version of manuscript: all authors. Approved the final version of the manuscript on behalf of all authors: Yao. Statistical analysis: Zhang, Lin, Wang, Qin. Administrative/technical/material support: Yao, Zhuang, Lin, Qin, Wu. Study supervision: Yao, Zhuang, Wu.

\section{Correspondence}

Cheng-Jun Yao, Glioma Surgery Division, Department of Neurologic Surgery, Huashan Hospital, Shanghai Medical College, Fudan University, 12\# Wulumuqi Zhong Rd., Shanghai 200040, China. email: chengjun_yao@126.com. 